\title{
LVI. Hydrometers of total immersion
}

\section{Arthur W. Warrington M.Sc.}

To cite this article: Arthur W. Warrington M.Sc. (1899) LVI. Hydrometers of total immersion, Philosophical Magazine Series 5, 48:295, 498-506, DOI: 10.1080/14786449908621444

To link to this article: http://dx.doi.org/10.1080/14786449908621444

$$
\text { 曲 Published online: } 08 \text { May } 2009 .
$$

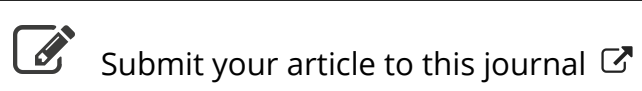

\footnotetext{
Џll Article views: 3
}

Q View related articles ¿ 
weight for the latter, the difference in the elastic properties is not so easily explained in that way. The diffusibility of canesugar is to that of white of $\mathrm{egg}$ as 7 to 1 . If these rates of diffusion are representative of the general properties of the two substances, we might expect to find a proportional double diffraction in solutions of eane-sugar. A marked effect of double refraction is found with 1 part of gelatine in 10,000 parts of solution. In a solution of cane-sugar containing 6000 parts in 10,000 no such effect could be found.

Even in the most dilute solutions of gelatine there must be a structure similar to that of solid jellies. When a small stress is applied, a strain arises in this structure which renders it double-refracting. When we go beyond the limit of elasticity this structure breaks down; and what we then have is probably a secondary effect, caused by the parts of a substance slipping over one another. It may be this secondary effect which has been studied in researches on this subject. It is easy to see how, after this breaking down reaches a maximum, the double refraction should increase as the velocity of rotation becomes greater, as is the case in the oils and in solutions of non-gelatinizing colloids. We can also conceive of conditions in which the amount of double refraction is not a function of the speed, as was found to be true with 4 grams in a litre at $27^{\circ}$.

That the rotation should change sign remains yet unexplained, but that it does so when a sufficiently high speed is reached cannot be doubted from the regularity with which it appeared.

My sincere thanks are due to Professor Brace, who has directed this investigation.

University of Nebraska, May 1899.

LVI. Hydrometers of Total Immersion. By Artrhur W. Warrington, M.Se.*

THE principal inconvenience of weighing in water arises from the fact that the fine suspension-thread is not uniformly moistened; the meniscus of the water changes its form and deadens the oscillations of the balance very rapidly.

F. Kohlrausch (Ann. der Phys. und Chem. vol. lvi. p. 187) finds that if the platinum suspension-wire be first covered electrolytically with platinum-black and then heated for a short time in a small bunsen-flame until it turns grey, it becomes uniformly and completely moistened when immersed * Communicated by the Author. 
in water. Using a thread so prepared, the oscillations of the balance are almost as uniform as in the air. P. Chappuis (Adjoint au Bureau International des Poids et Mesures) writes: "J'ai fait de nombreuses pesées hydrostatiques dans ces conditions et j'ai pu me convaincre de l'excellence de ce procédé qui donne une plus grande précision aux pesées hydrostatiques."

The present paper discusses a method of determining specific gravities which may in special cases be more convenient than the above, and is, I venture to think, quite as accurate.

Specific gravities are, by this method, determined by hydrometers which, when used, are totally immersed in the liquid.

Fig. 1.

Fig. 2.
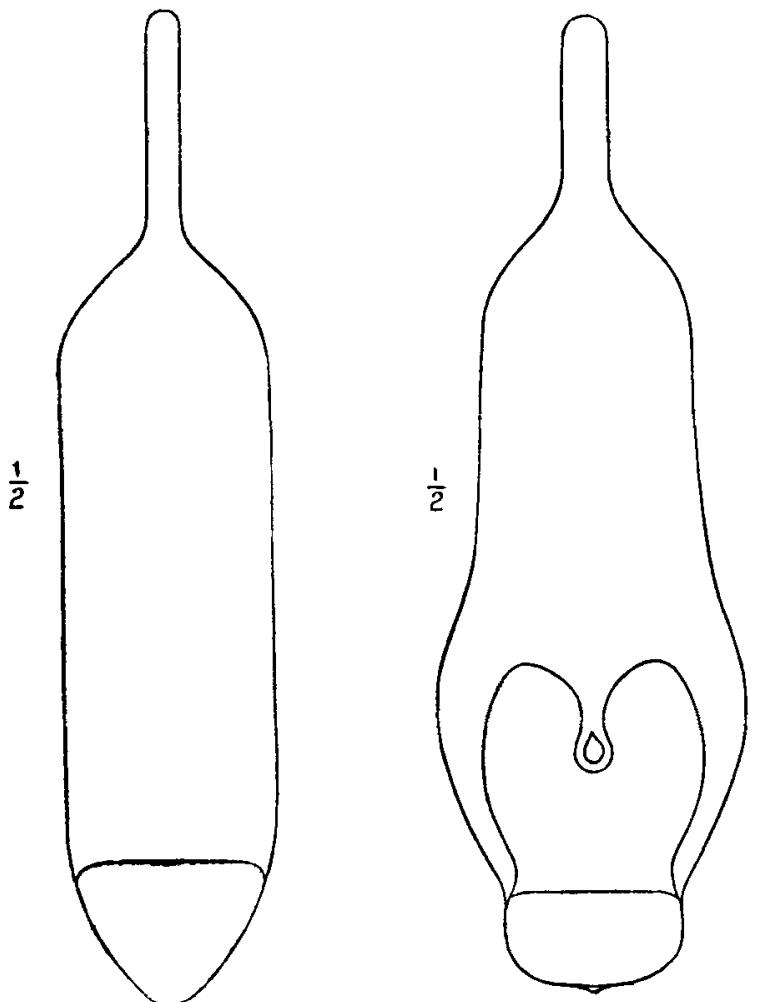

Fig. 1 shows the form used for liquids. By slipping smali ring-shaped platinum weights over the stem, the specific gravity of the hydrometer is made to approximate to that of 
the liquid ; the temperature of the latter is then allowed to change very slowly until a point is reached at which no difference can be detected between the specific gravities of the liquid and of the hydrometer.

Fig. 2 shows the form used for solids. Determinations are made in which the instrument has the same specific gravity as water (1) loaded with mercury alone, (2) loaded with the solid and mercury, care being taken that the temperature in both determinations is about the same.

The advantages claimed are:-

1st. That the method is exceedingly sensitive.

2nd. That the method is applicable within a considerable range of temperature without appreciable change in accuracy. My experiments ranged from $3^{\circ} .5$ to $35^{\circ}$.

3rd. All the weighings are made in air and under conditions which permit of repetition.

4th. The elimination of the use of a suspending thread should be beneficial.

For liquids the results are accurate to 1 in $1,000,000$; for solids to 1 in 100,000 .

I have used the proposed method to determine :-

(1) The coefficient of expansion of a hydrometer made of normal Jena glass.

(2) The temperature at which the density of water is at it: maximum.

(3) The specific gravity of a piece of quartz.

The Apparatus.

To obtain and maintain the required temperature the experiments were performed in an incubator. The incubator was made of steel plate, coated with lead, furnished with a double-walled isolated chamber and having two doors made of the best plate-glass. The outside of the inculator was covered with felt, and the bottom, which was funnel-shaped, was made of copper.

The top of the incubator was perforated with holes, to admit of the thermometer, the connecting rods of the stirrer, and a contrivance to clamp the hydrometer while stirring.

The stirrer was made of a flattened ring of silver, and was connected by two vertical rods to a cord which passedd round a pulley screwed into the ceiling.

Through the centre hole in the roof of the incubator passed a tube open at both ends and joined at its lower end to an inverted glass funnel. Before stirring the liquid the funnel was lowered until the hydrometer was clamped between it and the bottom of the beaker. When it was desired to 
liberate the hydrometer the funnel was raised, but only so far that its rim might catch the stem of the hydrometer should it attempt, as it invariably did at first, to float to inconvenient quarters of the beaker.

Besides the standard thermometer [11806] which was placed in the water, two other trustworthy thermometers were placed in such positions that they indicated the temperatures of the upper and lower strata of air in the chamber. A mirror was placed at the back of the air-chamber to reflect the light so as to facilitate the readings of the standard thermometer.

The position of the mercury column in the thermometer was read by means of a microacope furnished with a micrometer eyepiece.

Fig. 3 (p. 502) gives a section of the apparatus.

With this apparatus it was possible to attain any temperature between the atmosphere and $40^{\circ} \mathrm{C}$., and to regulate it so that it could be made to rise as slowly as $0^{\circ} .1$ per hour.

By allowing the final adjustment of temperature to take place with extreme slowness, and by frequent and effectual stirring, the prejudicial influence of convection-currents can be reduced to a negligible minimum. The best possible proof of the absence of convection-currents is furnished by the hydrometer itself, which, in the experiments described, often for minutes, remained poised in the middle of the water.

Generally at the time of reading the temperature was apparently constant. Several successive readings were often made which yielded identical results. In the neighbourhood of the thermometer the temperature seemed to change only at the time of stirring, remaining afterwards, for a time at least, stationary.

A glass envelope subjected to a change of temperature does not for some time assume its final volume corresponding to the new temperature. This interval of time is much greater when the temperature is lowered than when it is raised. Hence all the experiments were made at temperatures higher than that at which the hydrometer had been for some time previonsly, and no final reading was made until the hydrometer had been exposed to within a few tenths of a degree of that temperature for two or three hours.

\section{Method of carrying out an Experiment.}

Distilled water was poured into a beaker made of Jena glass and having a capacity of $3000 \mathrm{c}$. c. The water was then heated to within a degree of the required temperature, and Phil. Mag. S. 5. Vol. 48. No. 295. Dee. 1899. $2 \mathrm{~N}$ 
Fig. 3.

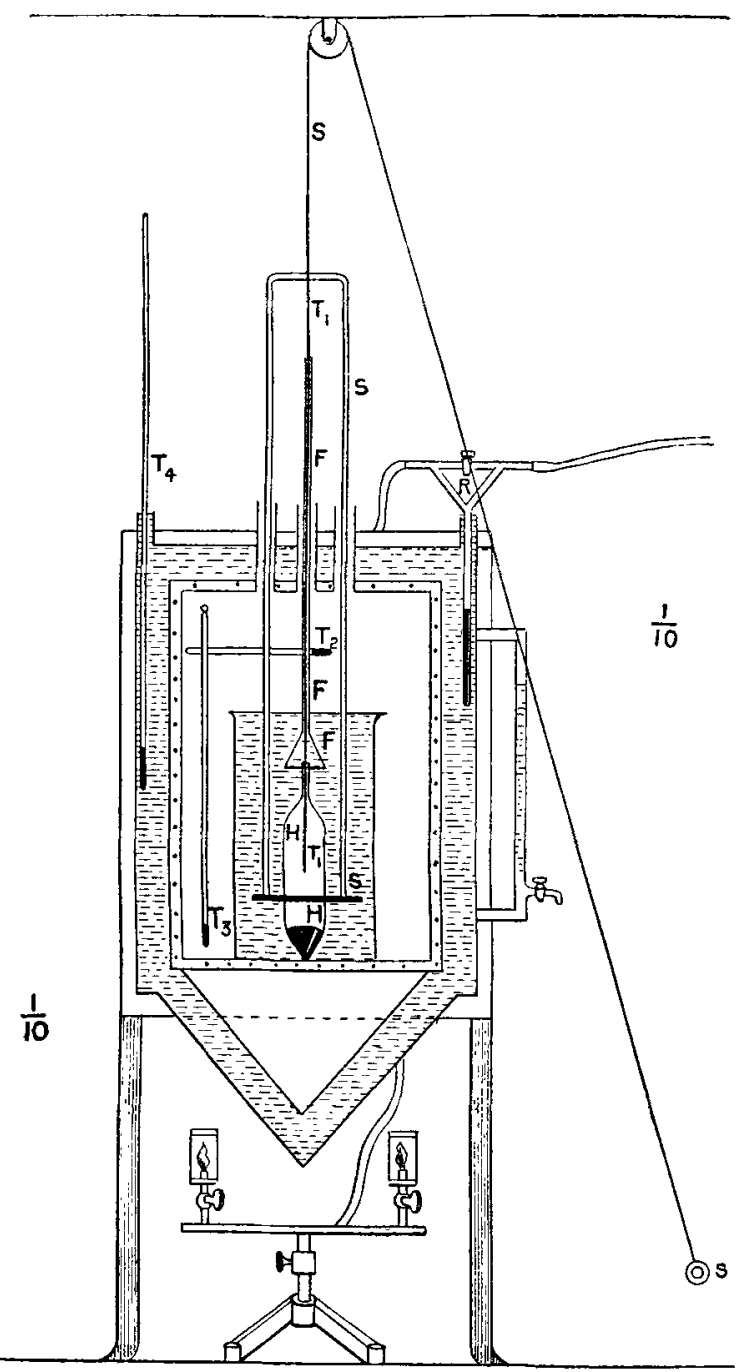

$\mathbf{H}=\mathbf{H y d r o m e t e r . ~}$

$\mathbf{T}_{1}=$ Principal Thermometer (Tonnelot). $\mathrm{F}=\mathrm{Funnel}$ and connecting rod. $\mathrm{T}_{2}, \mathrm{~T}_{3}, \mathrm{~T}_{4}=$ Auxiliary Thermometers. $\mathbf{S}=$ Stirrer apparatus. $\mathbf{R}=$ Gas Regulator. 
the hydrometer, wbich was only handled by the top of the stem

(For Liquids)

was removed from its wrappings, wiped with a clean handkerchief, and lowered slowly into the water. The platinum-ring weights to the calculated amount were boiled in distilled water and then picked up, one at a time, by a pair of ivorytipped forceps, and placed over the stem of the hydrometer.
(For Solids)

with its contents of nercury alone, or mercury and quartz, was slowly lowered into the water. Any small bubble of air on the surface of the mercury was removed by a glass pipette diawn out to a capiliary point.

The beaker and contents were then transferred to the incubator, and the hydrometer clamped into position by lowering the funnel. The stirrer was lowered to the bottom of the beaker, the thermometers put into their places, the doors closed, and the microscope fixed in position.

A preliminary experiment with the temperature changing somewhat quickly was then generally made to ascertain approximately at what temperature the hydrometer and the water had the same density.

Each observation was made as follows :-

1. The liquid was stirred.

2. The hydrometer was liberated by raising the funnel.

3. The thermometer was read. When the exact point was being reached, the readings were made with the divisions both in front and behind the mercury column.

4. The hydrometer was observed to see whether it tended to rise, to sink, or to occupy a position in the centre of the liquid.

Observations were repeated with the temperature rising or falling until no difference could be observed between the specific gravities of the hydrometer and water, or until two temperatures, differing only by a few thousandths of a degree, had been obtained at one of which the hydrorneter was heavier and at the other lighter than the water.

When the point of identical density was being approached, slight motions were imparted to the hydrometer by moving the funnel sideways or raising the stirrer, in order that the tendency of the hydrometer to sink or rise might not be masked by its touching the side of the funnel.

To repeat the experiment it was only necessary, if working with a rising temperature, to open the incubator-doors and waft in a little of the cooler atmospheric air, until the temperature of the water in the beaker fell a few hundredths of a $2 \mathrm{~N} 2$ 
degree. The doors were then closed and another experiment made.

Any mist which might form on the inside surface of the inner glass door was dissipated by cautiously warming the outside surface with a spirit-lamp.

Experiments 1 to 23 inclusive were made with a falling thermometer. The fall of the thermomster appeared in all cases to be perfectly uniform.

Experiment 39 is given in detail in Appen lix E.

\section{Determination of the Coefficient of Expansion of Normal Jena Glass.}

The hydrometer designed for liquids was made in July 1897 of normal Jena glass, and is weighted with mercury. It is $202 \mathrm{~mm}$. long and $41.5 \mathrm{~mm}$. diameter in its widest part. It is depicted in fig. 1 .

Its weight, as determined from the data given in Table I., is $160 \cdot 2150 \dot{b} \pm 0 \cdot 000077$ grammes.

If $\mathrm{W}$ be the weight in vacuo of the hydrometer itself and $v$ the weight in vacuo of the platinum which must be added just to submerge the instrument in water the specific gravity of which is $\rho$, then

$$
\mathrm{V}+v=\frac{\mathrm{W}+w}{\rho}, \quad \therefore \mathrm{V}=\frac{\mathrm{W}+w-\rho v}{\rho},
$$

in which $V=$ the volume of the hydrometer itself,

and $\quad v=$ the volume of the added platinum.

The values for $v$ were determined with such care that they were probably correct to $0.02 \mathrm{mg}$.

Six determinations (see Table II.) gave $21 \cdot 450 \pm 0.002$ as the mean specific gravity of the platinum used. Owing to the high specific gravity of platinum and the very small quantity of it used, $v$ can be calculated with sufficient accuracy to admit of the determination of $V$ in the above equation.

The volume of the hydrometer at $0^{\circ}$ when the first experiment was made was 161.92982 cub. centim.

Experiments made at approximately the same temperature, but on different days, proved not only that the volume of the hydrometer was continually contracting, but also furnished data for a quantitative estimation of the rate of contraction.

The mean expansion of the hydrometer between $0^{\circ}$ and $40^{\circ}$ for one degree is about 0.0039 c. c. Hence if the volume of the hydrometer at temperature $t$ be $V_{t}$, then its volume at temperature $t_{1}$, when $t=t_{1} \pm 0^{\circ} \cdot 2$, on the same day, is given 
without sensible error by the formula

$$
\mathrm{V}_{t_{1}}=\mathrm{V}_{t}-0.0039\left(t-t_{1}\right) \ldots . . .
$$

Assuming that the volume of the hydrometer may be expressed as a function of the time in the form

$$
\mathrm{V}_{t_{1} \Delta}=\mathrm{V}_{t_{1}}+a \Delta+b \Delta^{2}, . . . . .
$$

when $V_{t_{1} \Delta}$ is its volume at temperature $t_{1}$ and $\Delta$ is the time in days, then on some other day $\Delta_{1}$ at the same temperature its volume will be

$$
V_{t_{2} \Delta_{1}}=V_{t_{1}}+a \Delta_{1}+b \Delta_{1}^{2} ; . . . . .
$$

subtracting equation (3) from equation (2) we get

$$
\mathrm{V}_{t_{1} \Delta}-\mathrm{V}_{t_{1} \Delta_{1}}=a\left(\Delta-\Delta_{1}\right)+b\left(\Delta^{2}-\Delta_{1}^{2}\right) . .
$$

Calculated by the method of least squares from every experiment which permitted the use of equation (4), the most probable value of $a$ is

and of $b$

$$
-0000,008,262,84
$$

$$
+0 \cdot 000,000,016,286 \text {. }
$$

Further, assuming that the volume of the hydrometer for any temperature $t$ can be found by the formula

$$
\mathrm{V}_{t}=\mathrm{V}_{0}+x t+y t^{2}
$$

and by means of the above values of $a$ and $b$ reducing all observations to the day on which the first experiment was made, the most probable values of $\mathrm{V}_{0}, x$, and $y$ are

which gives

$$
\begin{aligned}
& \mathrm{V}_{0}=161 \cdot 929816, \\
& x=+0 \cdot 003,839,9, \\
& y=+0 \cdot 000,001,881 ;
\end{aligned}
$$

$$
\mathrm{V}_{t}=\mathrm{V}_{0}\left[1+(23,714+11 \cdot 62 t) 10^{-9} t\right] \text {. }
$$

According to Thiesen and Scheel (Zeitschrift für Instrk. xii. p. 294, 1893) the linear coefficient of expansion of normal Jena glass is

$$
(7797+3 \cdot 64 t) 10^{-9}
$$

which would give a cubical coefficient of expansion of

$$
(23,391+10 \cdot 92 t) 10^{-9} \text {. }
$$

In Table III. the figures given in the last column but one 
are calculated from the formula

$$
\mathrm{V}_{t \Delta}=\mathrm{V}_{0}+a \Delta+b \Delta^{2}+x t+y t^{2},
$$

the values for $\mathrm{V}_{0}, a, b, x$, and $y$ being those given above.

In the last column of the table under $\delta$ are given the differences between the observed and calculated volumes of the hydrometer. The probable error of a single determination of the volume of the hydrometer is $\pm 0.000111 \mathrm{eub}$. centim.

The table includes every experiment made; none have been rejected.

\section{The Temperature at which the Density of Water is at a Maxinum.}

Professor Schuster, for whose kindness I am very deeply indebted, suggested that I might test the efficacy of the method for the determination of temperatures of maximum density. Experiments 24 to 32 inclusive were made with this object more especially in view, and are discussed in the following note by Mr. G. A. Schott, B.A., B.Sc., a colleague to whom I owe much for his valuable advice.

The data used by Mr. Schott are obtained from an earlier and slightly less exact calculation than that giren in this paper.

Note by Mr. G. A. Schorr, B.A., B.Sc.

The Determination of the Point of Maximum Density of a Liquid by the Hydrometer of Totai Immersion.

The volume of the hydrometer is supposed given at the temperature of the experiment, say $\mathrm{V}=\mathrm{A}^{\prime}+\mathrm{B}^{\prime} t+\mathrm{C}^{\prime} t^{2}$, and the probable errors of $A^{\prime}, B^{\prime}, C^{\prime}$ are supposed known.

The weight necessary to totally immerse the hydrometer in the neighbourhood of the point of maximum density is determined by a number of experiments, and expressed in the form $\mathrm{A}+\mathrm{B} t+\mathrm{C} t^{2}$.

Then the density of the liquid is

$$
\rho=\frac{\mathrm{A}+\mathrm{B} t+\mathrm{C}^{\prime} t^{2}}{\mathrm{~A}^{\prime}+\mathrm{B}^{\prime} t+\mathrm{C}^{\prime} t^{2}}=\frac{\mathrm{A}}{\mathrm{A}^{\prime}} \cdot \frac{1+\frac{\mathrm{B}}{\mathrm{A}} t+\frac{\mathrm{C}}{\mathrm{A}} t^{2}}{1+\frac{\mathrm{B}^{\prime}}{\mathrm{A}^{\prime}}+\frac{\mathrm{C}^{\prime}}{\mathrm{A}^{\prime}} t^{2}} .
$$

The temperature $\tau$ of maximum density is determined by the 